\title{
BUCKLING ANALYSIS OF HETERO-JUNCTION CARBON NANOTUBES
}

\author{
BOCKO Jozef ${ }^{1}$, LENGVARSKÝ Pavol ${ }^{1}$, ŠARLOŠI Juraj ${ }^{1}$ \\ ${ }^{I}$ Technical University of Košice, Faculty of Mechanical Engineering, Department of Applied Mechanics and \\ Mechanical Engineering, Letná 9, 04200 Košice, Slovak Republic, email: jozef.bocko@tuke.sk
}

\begin{abstract}
The buckling analysis of carbon nanotubes without and with hetero-junctions is described in this paper. The buckling behaviour was investigated by the finite element method and the carbon nanotubes were modelled as space frame structures. The results showed that the critical buckling force depends on the dimensions of carbon nanotubes. The critical buckling forces of hetero-junction carbon nanotubes are in range between critical buckling forces of carbon nanotubes of both used diameters with the same chiralities without hetero-junction.
\end{abstract}

KEYWORDS: Carbon nanotubes, Buckling, Hetero-junction, Beam element.

\section{Introduction}

The carbon nanotubes were discovered by Iijima in 1991 [1]. From the discovery of nanostructures, their mechanical, thermal and electrical properties have been widely studied [112]. The buckling behaviour of carbon nanotubes is often studied because of their relative dimensions - the nanotubes are relatively long with respect to their diameters and they have small wall thickness. On the buckling behaviour have effect dimension but also vacancies in the structure, boundary conditions and connection of carbon nanotubes. The buckling behaviour of nanotubes was studied in papers [2-4].

In this paper the buckling analysis of single-walled carbon nanotubes without and with hetero-junctions is described. The carbon nanotubes with two chiralities (armchair and zigzag) are modelled using finite element method. All degrees of freedoms of movement are constrained on the one end of nanotube and axial loading is applied to the second end. The computed critical buckling forces are represented in graphs.

\section{Modelling of carbon nanotubes}

The carbon nanotubes are modelled using the beam elements. The input properties are obtained by making connection between molecular mechanics and continuum mechanics. In the molecular mechanics we know interatomic interactions (Fig. 1) as bond stretching, bond angle variation, dihedral angle torsion and out of plane torsion. In the continuum mechanics these interactions can be represented by the beam elements loaded by pure tension, bending and torsion. This connection can be expressed through relations

$$
\begin{gathered}
U_{r}=\frac{1}{2} k_{r}\left(r-r_{0}\right)^{2}=\frac{1}{2} k_{r}(\Delta r)^{2}, \\
U_{\theta}=\frac{1}{2} k_{\theta}\left(\theta-\theta_{0}\right)^{2}=\frac{1}{2} k_{\theta}(\Delta \theta)^{2},
\end{gathered}
$$




$$
U_{\tau}=U_{\phi}+U_{\theta}=\frac{1}{2} k_{\tau}(\Delta \phi)^{2},
$$

where $k_{r}, k_{\theta}, k_{\tau}, \Delta r, \Delta \theta, \Delta \phi$ are the bond stretching force constant, bond angle bending force constant, torsional resistance, the bond stretching increment, the bond angle variation and the angle variation of bond twisting, respectively [5-7].

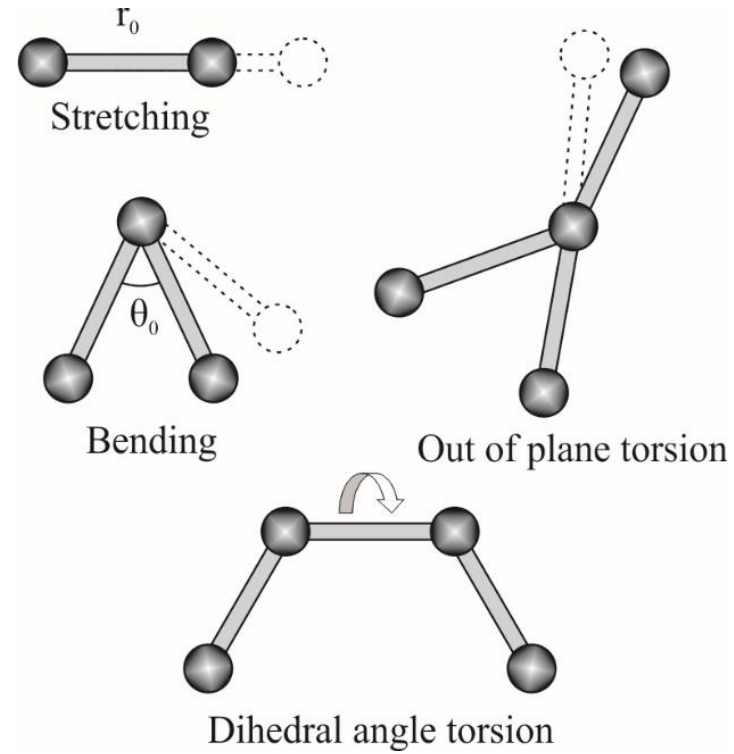

Fig. 1 Interatomic interactions between carbon atoms

The elastic moduli of beam elements are evaluated from mechanical considerations relations between the sectional stiffness parameters in structural mechanics and the force constants in molecular mechanics. In case the sections of beams representing carbon-carbon bonds are assumed to be identical and circular, the moments of inertia are $I_{x}=I_{y}=I$. Three stiffness parameters $E A, E I$ and $G J$ are obtained from this assumption and from linkage among the energy terms in molecular mechanics and continuum mechanics. It is possible to write relations

$$
\begin{gathered}
U_{A}=\frac{1}{2} \int_{0}^{L} \frac{N^{2}}{E A} d L=\frac{1}{2} \frac{N^{2} L}{E A}=\frac{1}{2} \frac{E A}{L}(\Delta L)^{2}, \\
U_{M}=\frac{1}{2} \int_{0}^{L} \frac{M^{2}}{E I} d L=\frac{2 E I}{L} \alpha^{2}=\frac{1}{2} \frac{E I}{L}(2 \alpha)^{2}, \\
U_{T}=\frac{1}{2} \int_{0}^{L} \frac{T^{2}}{G J} d L=\frac{1}{2} \frac{T^{2} L}{G J}=\frac{1}{2} \frac{G J}{L}(\Delta \beta)^{2},
\end{gathered}
$$

where $U_{A}$ is the strain energy of a uniform beam of length $L$ subjected to axial force $N, \Delta L$ is axial elongation, $U_{M}$ is the strain energy of a uniform beam under bending moment $M, \alpha$ is the rotational angle at the ends of the beam, $U_{T}$ is the strain energy of a uniform beam under tension $T$ and $\Delta \beta$ is the relative rotation between the ends of the beam [8,9].

Comparison of equations (1)-(3) and (4)-(6) leads to equations

$$
\frac{E A}{L}=k_{r}, \quad \frac{E I}{L}=k_{\theta}, \quad \frac{G J}{L}=k_{\tau} .
$$


On the basis of these equations a beam element (Fig. 2) is created and its elastic properties for further computations are

$$
d=4 \sqrt[4]{\frac{k_{\theta}}{k_{r}}}, \quad E=\frac{k_{r}^{2} L}{4 \pi k_{\theta}}, \quad G=\frac{k_{r}{ }^{2} k_{\tau} L}{8 \pi k_{\theta}^{2}},
$$

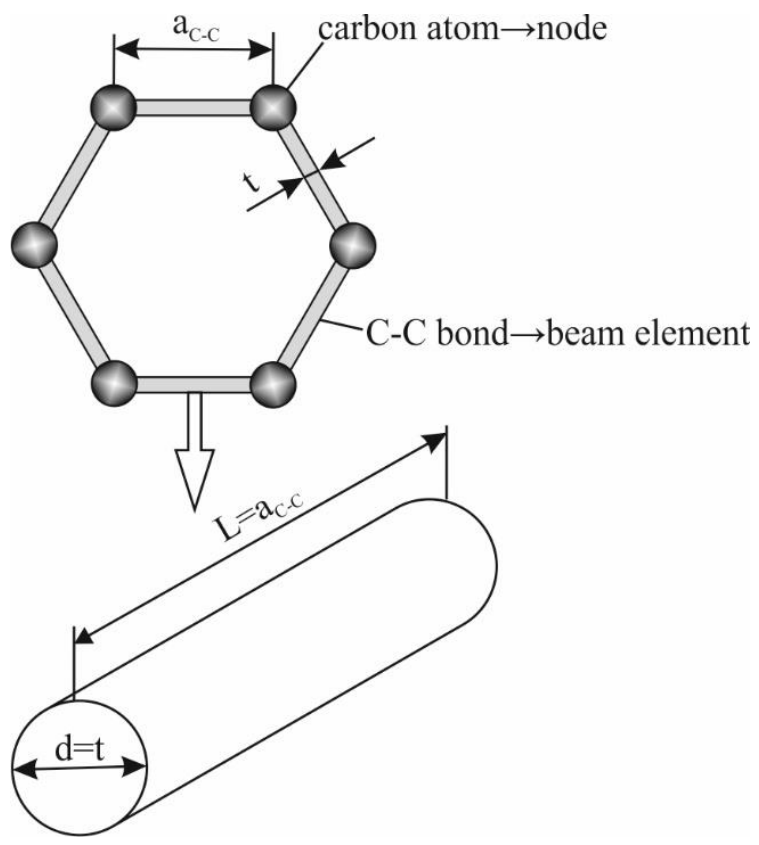

Fig. 2 The dimensions of carbon hexagon made from beam elements

where constants $k_{r}, k_{\theta}, k_{\tau}$, L are $k_{r}=6.52 \times 10^{-7} \mathrm{~N} \mathrm{~nm}^{-1}, k_{\theta}=8.76 \times 10^{-10} \mathrm{~N} \mathrm{~nm} \mathrm{rad}^{-1}$, $k_{\tau}=2.78 \times 10^{-7} \mathrm{~N} \mathrm{~nm} \mathrm{rad}^{-2}$ and $L=a_{C-C}=0.1421 \mathrm{~nm} \mathrm{[10]}$.

From the equations (8) the diameter $d=0.147 \mathrm{~nm}$, elastic modulus $E=5.4875 \mathrm{TPa}$ and shear modulus $G=0.871 \mathrm{TPa}$ can be computed and used for modelling the beam elements.

\section{Buckling of hetero-junction carbon nanotubes}

The buckling analysis is performed for carbon nanotubes without and with hetero-junctions. The armchair $(\mathrm{n}, \mathrm{n})$ and zigzag $(\mathrm{n}, 0)$ chirality is used for modelling carbon nanotubes. The length of the carbon nanotubes varies from $3 \mathrm{~nm}$ to $48 \mathrm{~nm}$. The used chiralities and diameters of carbon nanotubes are given in Table 1. The hetero-junction carbon nanotubes are made as the connection of the two carbon nanotubes (Fig. 3) with the same chirality and with the different diameter (Table 2).

Diameter of carbon nanotubes is computed from relation

$$
D=\frac{a_{0}}{\pi} \sqrt{n^{2}+m^{2}+n m},
$$

where $a_{0}=0.246 \mathrm{~nm}$.

Tab. 1 Chiralities and diameters of modelled carbon nanotubes

\begin{tabular}{|l|c|c|c|c|c|c|}
\hline Chirality (n,m) & $(6,0)$ & $(8,0)$ & $(10,0)$ & $(4,4)$ & $(5,5)$ & $(6,6)$ \\
\hline Diameter (nm) & 0.4701 & 0.6268 & 0.7834 & 0.5428 & 0.6785 & 0.8142 \\
\hline
\end{tabular}


Tab. 2 Modelled hetero-junctions of carbon nanotubes

\begin{tabular}{|l|l|l|l|}
\hline Chirality (n,m) & $(6,0)-(8,0)$ & $(6,0)-(10,0)$ & $(8,0)-(10,0)$ \\
\hline Diameter (nm) & $0.4701-0.6268$ & $0.4701-0.7834$ & $0.6268-0.7834$ \\
\hline Chirality $(\mathbf{n}, \mathbf{m})$ & $(4,4)-(5,5)$ & $(4,4)-(6,6)$ & $(5,5)-(6,6)$ \\
\hline Diameter (nm) & $0.5428-0.6785$ & $0.5428-0.8142$ & $0.6785-0.8142$ \\
\hline
\end{tabular}

c)

Fig. 3 Carbon nanotube a) with zigzag chirality $(6,0)$, b) with zigzag chirality $(10,0)$ and c) hetero-junction with zigzag chirality $(6,0)-(10,0)$

The carbon nanotubes are modelled using beam elements with six degrees of freedom at the node, with material properties and length mentioned above. The beam elements represent the interatomic interactions and the nodes represent the carbon atoms. The finite element analysis is performed for nanotubes fixed on the one end (end with bigger diameter for the heterojunction carbon nanotubes) and the second one is loaded by axial force. The critical buckling forces for the all carbon nanotubes are computed by the finite element method. The all computed results are shown in Figs. 4-9. From these figures is clear that the critical buckling forces of nanotubes depend on the diameter and length. The magnitudes of critical buckling forces for hetero-junction carbon nanotubes intervene between magnitudes of critical buckling forces of carbon nanotubes with the same chirality as both ends of these tubes.

\section{CONCLUSION}

In this work, a finite element method was used for modelling and simulation of the buckling behaviour of carbon nanotubes. The carbon nanotubes were modelled as space frame structures, where the interatomic interactions were represented by the beam elements and the carbon atoms were represented by the nodes of beam elements. The input properties for beam elements were obtained by creating a connection between molecular and continuum mechanics. The two types of carbon nanotubes were modelled: carbon nanotubes with and without hetero-junction. For the complexity, the carbon nanotubes with armchair and zigzag chirality were modelled too, but the effect of chirality on the buckling behaviour is negligible from the point of view of prevailing dimensions (diameter and length) of nanotube. The boundary nodes of the one end of nanotube were constrained and the ones on the second end were loaded by axial force. The critical buckling forces were obtained by the finite element method and results were represented in graphs. The critical buckling forces for hetero-junction carbon nanotubes lie between critical buckling forces of carbon nanotubes with the same chirality of both ends of these tubes. 


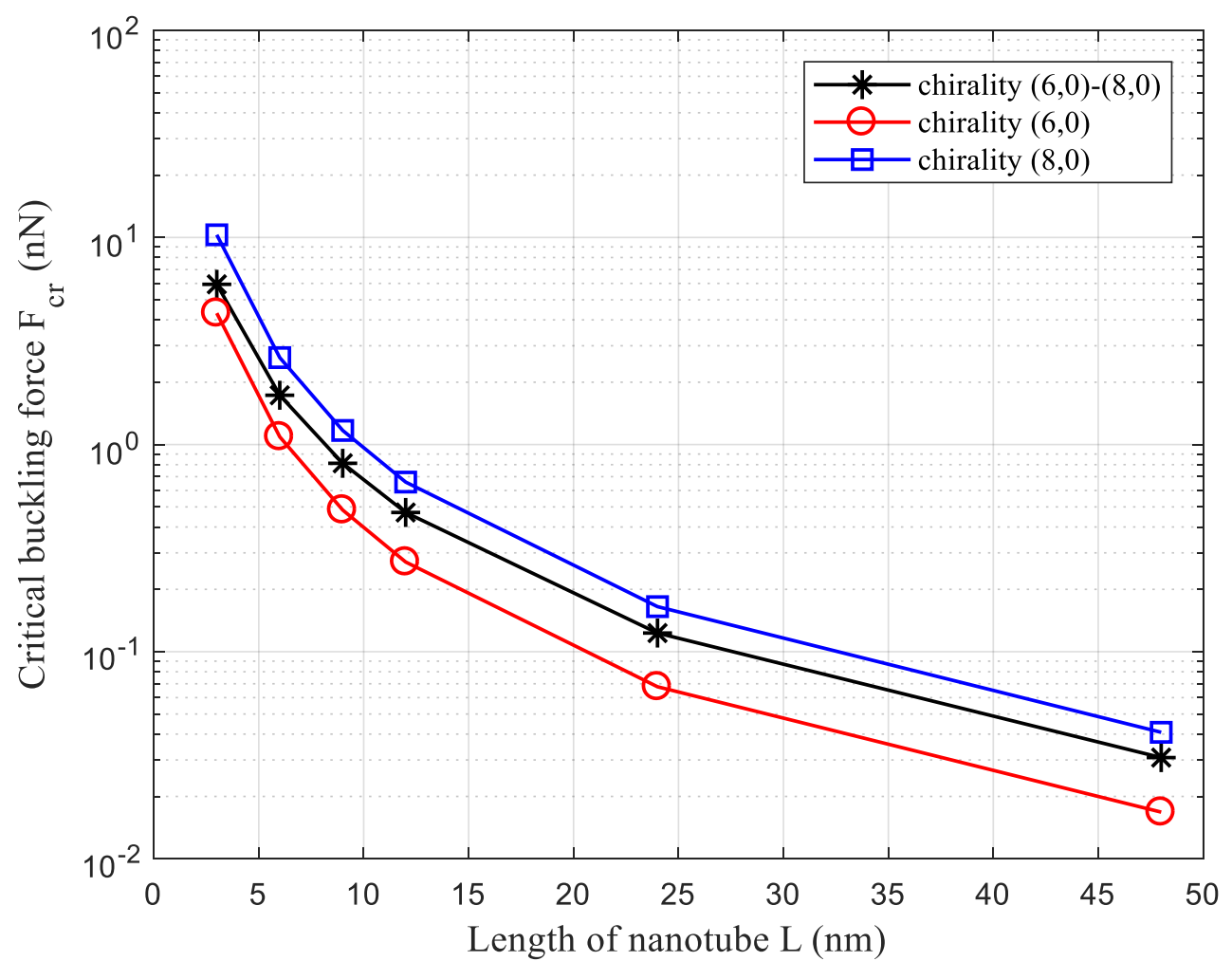

Fig. 4 Critical buckling forces for zigzag carbon nanotubes

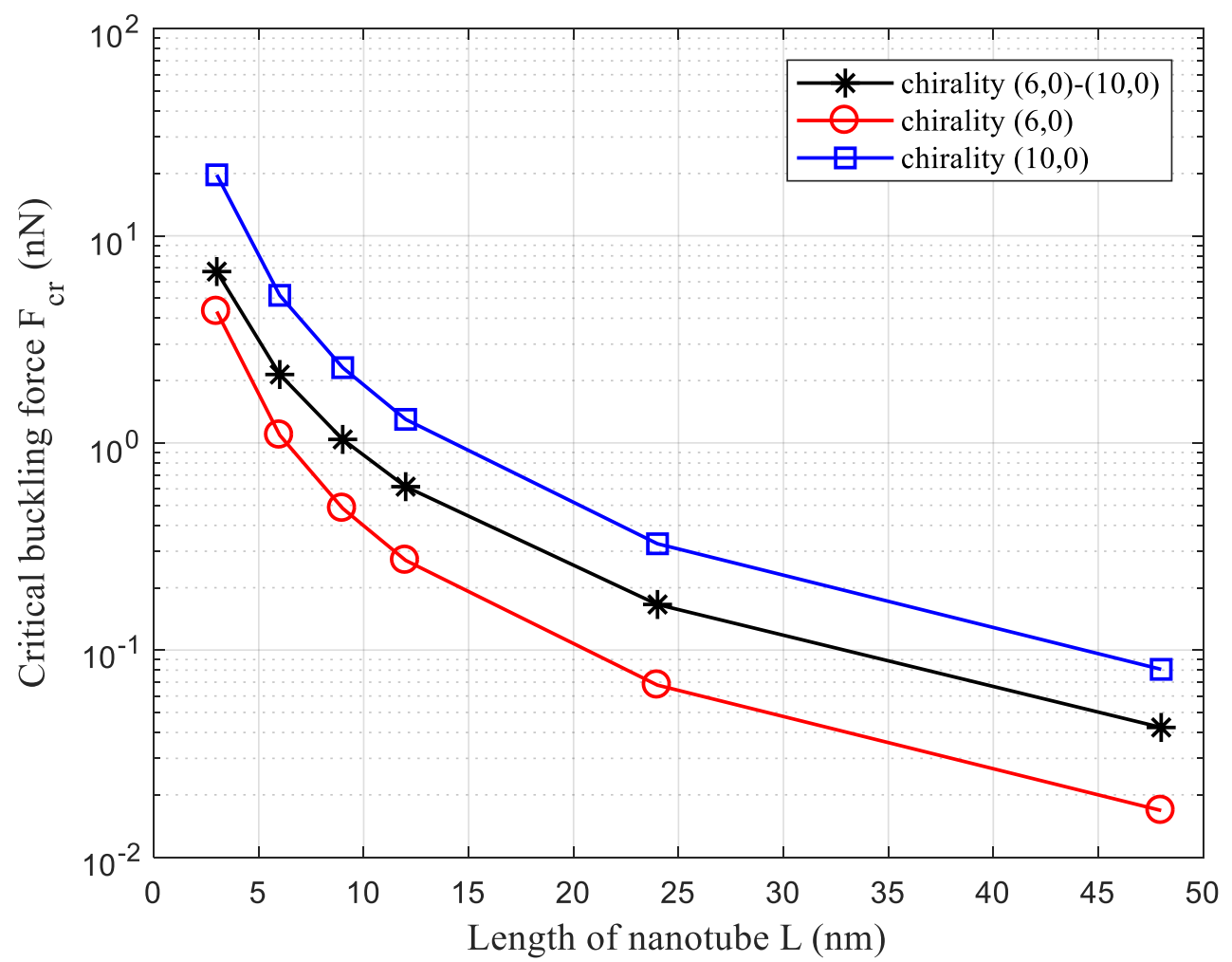

Fig. 5 Critical buckling forces for zigzag carbon nanotubes 


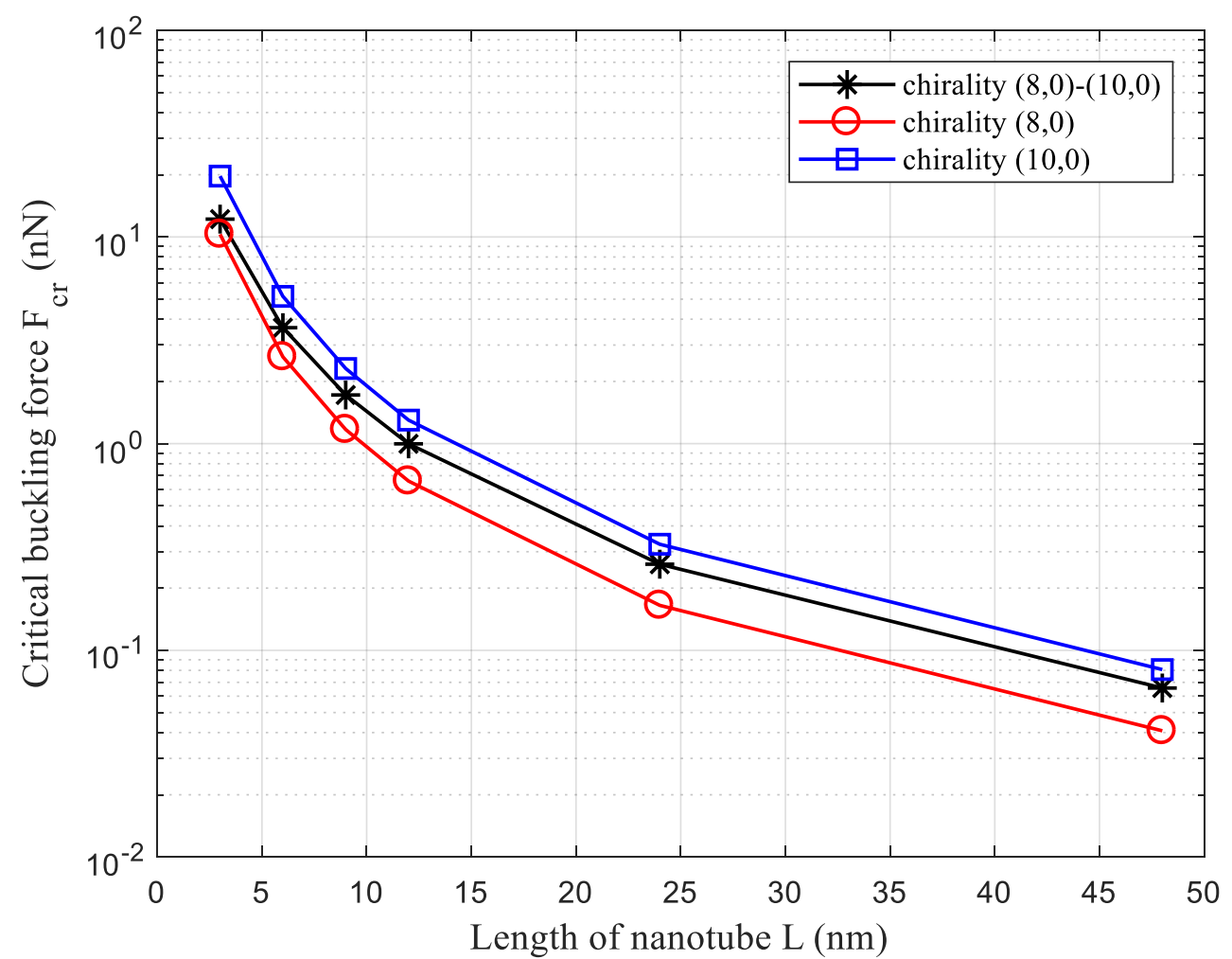

Fig. 6 Critical buckling forces for zigzag carbon nanotubes

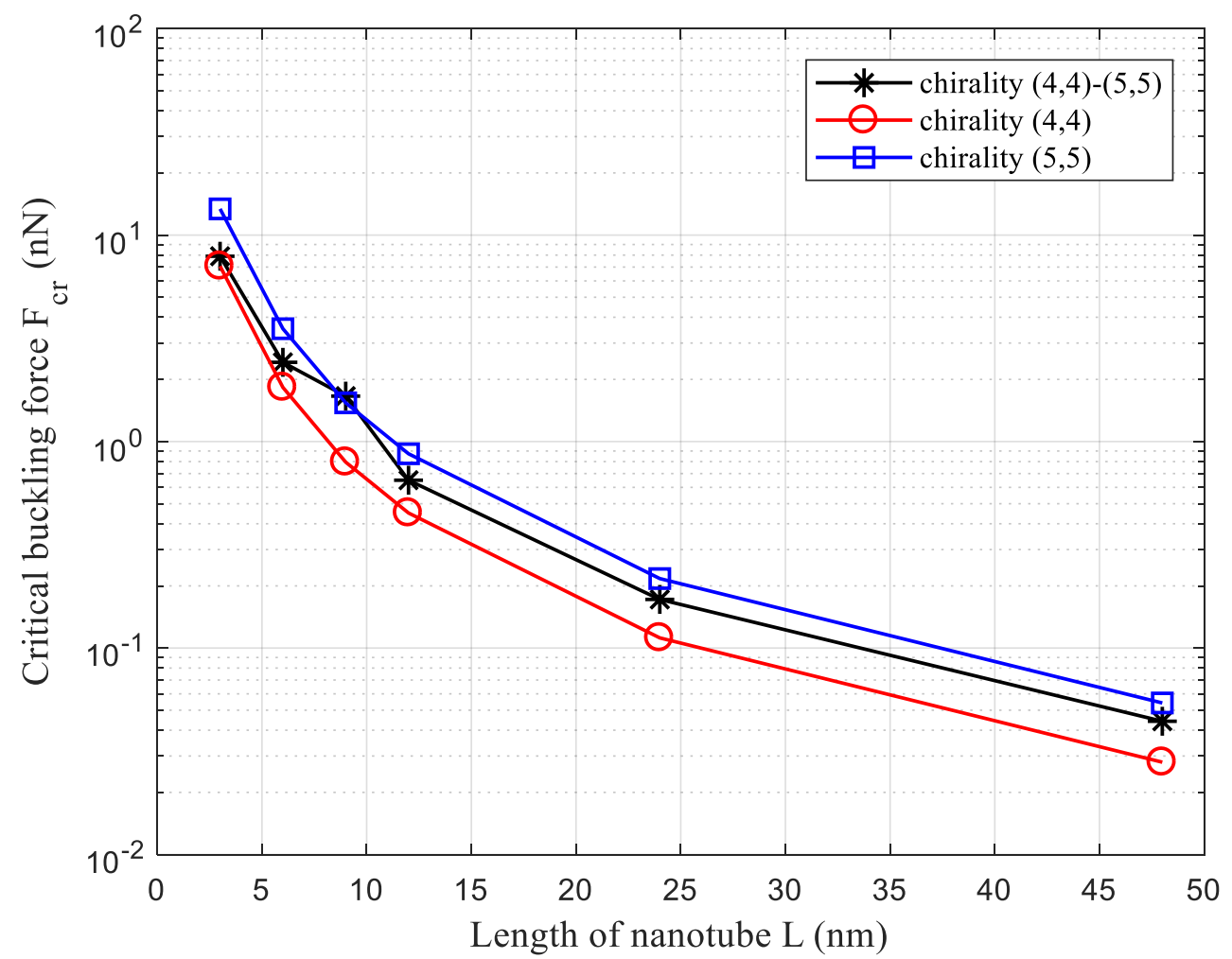

Fig. 7 Critical buckling forces for armchair carbon nanotubes 


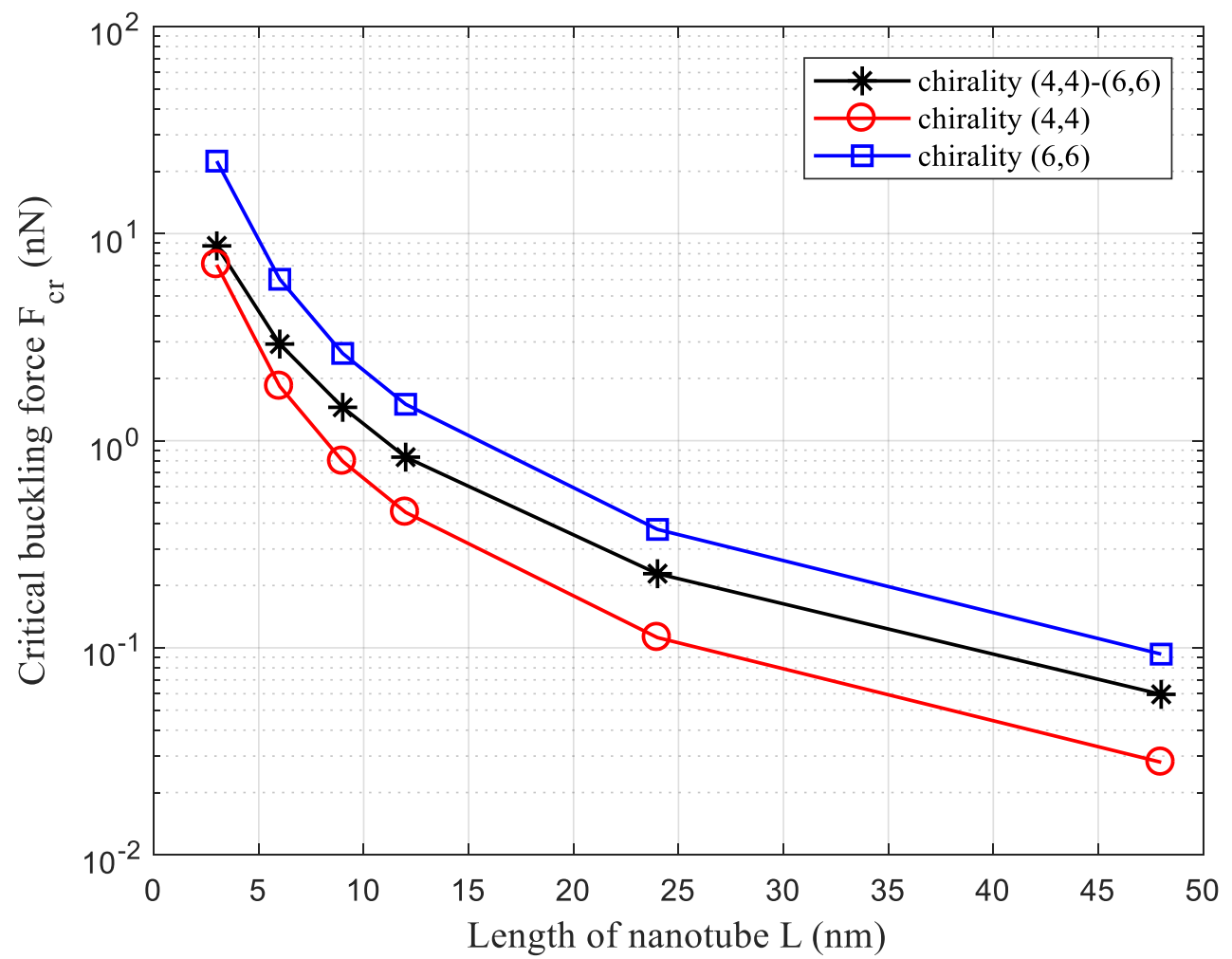

Fig. 8 Critical buckling forces for armchair carbon nanotubes

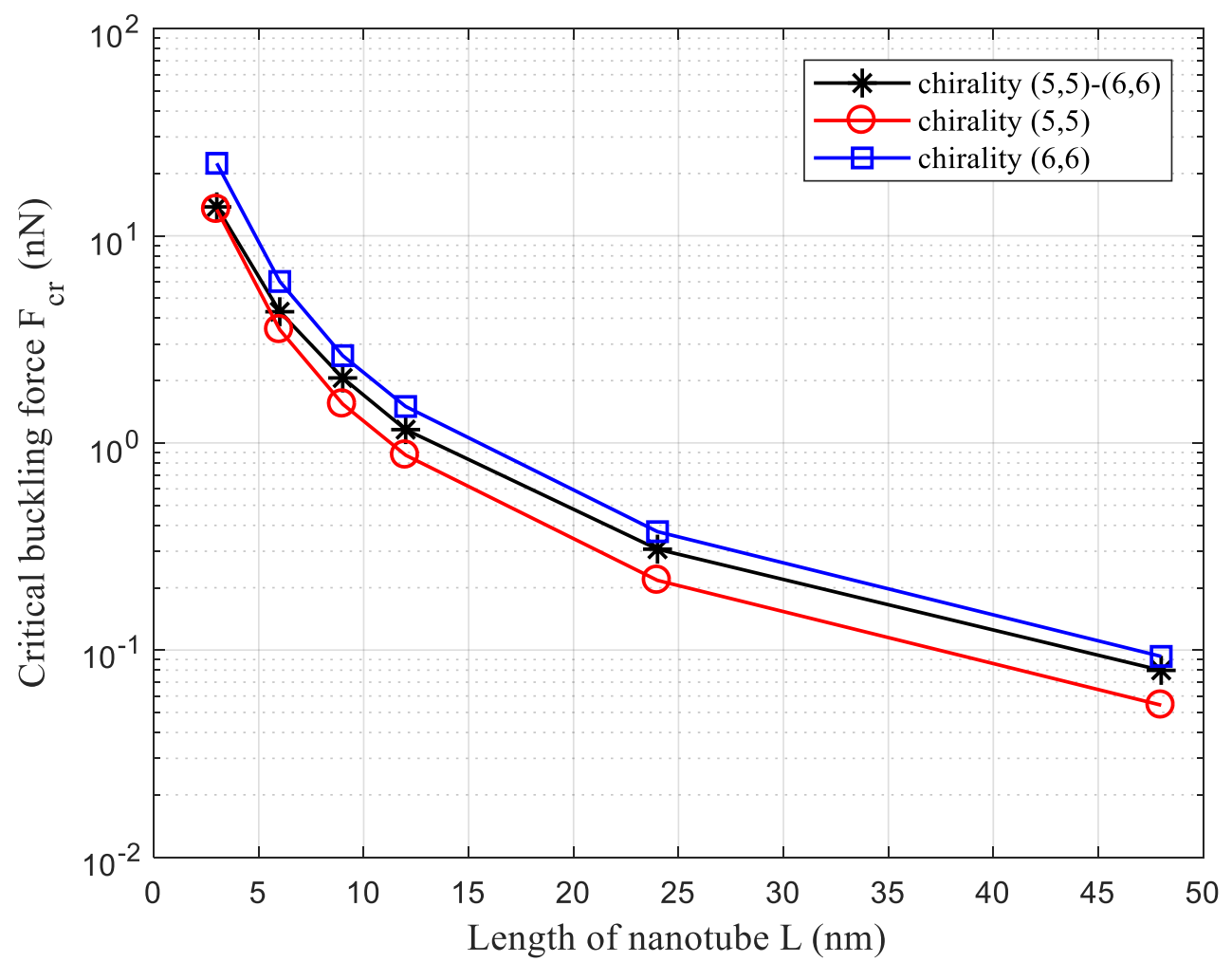

Fig. 9 Critical buckling forces for armchair carbon nanotubes 


\section{ACKNOWLEDGMENTS}

This research was supported by a grant from Slovak Grant Agency VEGA No. 1/0731/16 and VEGA 1/0355/18.

\section{REFERENCES}

[1] S. Iijima, Helical microtubules of graphitic carbon, Nat. 1991 (354), 56-58.

[2] A. Ghavamian, A. Öchsner. On the buckling behavior of perfect and atomically defective hetero-junction carbon nanotubes. Mech. Adv. Mater. Struc. 2017 (24), 1043 - 1057.

[3] S.K. Georgantzinos, G.I. Giannopoulos. Thermomechanical buckling of single walled carbon nanotubes by a structural mechanics method. Diamond Relat. Mater. 2017 (80), $27-37$.

[4] J. Bocko, P. Lengvarský. Buckling of single-walled carbon nanotubes with and without defects. J. Mech. Sci. Technol. 2017 (4), 1825 - 1833.

[5] D.W. Brenner. Empirical potential for hydrocarbons for use in simulating the chemical vapor deposition of diamond films. Phys. Rev. B. 1990 (42), 9458.

[6] A. Choudhury, S. Mondal, S. Sarkar. Effect of lamination angle and thickness on analysis of composite plate under thermo mechanical loading. Journal of Mechanical Engineering - Strojnícky časopis 2017 (67), No. 1, 5 - 22.

[7] W.D. Cornell, P. Cieplak, C.I. Bayly. A second generation force-field for the simulation of proteins, nucleic-acids, and organic-molecules. J. Ame. Chem. Soc. 1995 (117), 5179 $-5197$.

[8] A. K. Rappe, C.J. Casewit, K.S. Colwell. A full periodic-table force-field for molecular mechanics and molecular dynamics simulations. J. Ame. Chem. Soc. 1992 (114), 10024 -10035 .

[9] Ch. Li, T.W. Chou. A structural mechanics approach for the analysis of carbon nanotube. Int. J. Solids Struc. 2003 (40), 2487 - 2499.

[10] K.I. Tserpes, P. Papanikos. Finite element modelling of single-walled carbon nanotubes. Compos. Part B Eng. 2005 (36), $468-477$.

[11] V.K. Patel, K. Rani. Mechanical and wear properties of friction stir welded 0-6Wt\% nAl2O3 reinforced Al-13Wt\%Si composites. Journal of Mechanical Engineering Strojnícky časopis 2017 (67), No. 1, 77 - 86.

[12] E. Marenić, A. Ibrahimbegovic, J. Sorić, P.A. Guidault. Homogenized elastic properties of graphene for small deformations. Materials 2013 (6), $3764-3782$. 